\title{
Stenasellus bragai n. sp., Crustacea Isopoda Asellota des eaux souterraines d'Espagne méridionale
}

\author{
par
}

Guy MAGNIEZ

\section{SUMMARY \\ Stenasellus bragai n. sp., Crustacea Isopoda Asellota from subterranean waters of Southern Spain}

This small species has been round in the underflow of Guadalquivir River (Jaèn Province). It belongs to the phyletic line of Stenasellus hreuili Racovitza, distributed in the underground waters of the Iberic Peninsula.

Origine du matériel: Cette nouvelle espèce a été capturée, en août 1972, par Monsieur Claude Bou, au cours de ses prospections des eaux souterraines d’Espagne méridionale, par le procédé des sondages tubés Bou-Rouch, dans le Guadalquivir, à environ $10 \mathrm{~km}$ en amont du confluent de celui-ci avec le Guadiana Menor (un peu en aval du barrage supportant la route de Torreperogil à Peal-de-Becerro, province de Jaèn), (renseignements aimablement communiqués par $\mathrm{M}$. Cl. Bou, que je remercie de la confiance qu'il me témoigne en mettant ce matériel à ma disposition).

La nouvelle espèce est dédiée au regretté Docteur José Maria Braga, de Porto, en raison de la contribution éminente qu'il a apportée à la connaissance des Asellotes d'eau douce et singulièrement des Sténasellides.

Lot capturé: $3 \hat{\circ}$ adultes de 3,$2 ; 2,9$ et $2,5 \mathrm{~mm} ; 1 \stackrel{+}{ }$ adulte à poche incubatrice vide de $3,2 \mathrm{~mm} ; 2$ adultes au repos génital de 3,4 et $3,3 \mathrm{~mm} ; 6$ jeunes de 2,$3 ; 1,9 ; 1,9 ; 1,8 ; 1,8$ et $1,7 \mathrm{~mm}$ et 2 pulli de $1,1 \mathrm{~mm}$, sans péréiopodes VII.

* Laboratoire de Biologie animale et générale, Université de Dijon, 6, Bd. Gabriel, 21000

Dijon, France. 
Caractères généraux: St. bragai $\mathrm{n}$. sp. est le plus petit Stenasellus connu, les plus petites formes décrites précédemment atteignant $5 \mathrm{~mm}$. Parmi les Sténasellides, on connaissait déjà la forme naine Magniezia studiosorum Sket des sources de Kedougou (Sénégal), tandis que le plus grand Stenasellidae, St. costai Lanza, Chelazzi et Messana de Somalie dépasse $25 \mathrm{~mm}$. La nouvelle espèce montre une nette adaptation à la vie psammique: tégument membraneux et peu induré, corps grêle (coefficient d'allongement de 5-6), appendices grêles et fragiles (fouet des $\mathrm{A} 1, \mathrm{~A} 2$, péréiopodes et uropodes cassés chez la plupart des individus), chétotaxie générale très réduite.

Céphalon subtrapézoïdal un peu plus large que long, à bord rostral légèrement concave et angles rostraux très atténués. Moitié antérieure du corps nettement moins large que la postérieure (du péréionite $\mathrm{V}$ au pléon) sauf chez la $q$ à poche incubatrice, dont les péréionites II, III et IV sont élargis. Régions pleurales des péréionites étroites et coxopodites II-VII libres et normalement développés (comme chez St. virei). Pléonites libres I et II relativement peu développés en longueur. Pléotelson allongé, à pointe caudale marquée (fig. 2).

Appendices céphaliques: Antennules avec jusqu'à 10 articles au fouet et 3 lames olfactives de 100-105 $\mu$ sur les distaux (5 articles et 1 L.O. seulement chez les jeunes de $1,9 \mathrm{~mm}$ ), (fig. 7).

Antenne avec squama bien développée sur le 3ème article de la hampe (fig. 4). Fouet d'une quinzaine d'articles chez un individu de $1,9 \mathrm{~mm}$.

Mandibule très recourbée, typique (fig. 15), avec palpe triarticulé très grand; processus inciseur et lacinie mobile normaux, chétotaxie réduite sur la partie molaire.

Maxillule normale, à lobe externe portant une douzaine de fortes épines barbelées distales de $30 \mu$ et des sétules sur les marges latérales; lobe interne avec 3 fortes tiges rameuses de $40 \mu$ et une soie simple moitié moins longue sur la marge distale; marge externe garnie de sétules.

Maxille trilobée normale. Lobe externe avec une rangée distale de 10 lames falciformes pectinées sur le bord interne et décroissant de l'extérieur

Planche I:

1. Dactylopodite du péréiopode 2 droit, du ồ de $2,9 \mathrm{~mm} ; \mathrm{e}=33 \mu$.

2. Région postérieure (segments thoraciques 7 et 8 , pléonites 1 et 2 , pléotelson et uropodes) d'un individu de $1,9 \mathrm{~mm} ; \mathrm{e}=200 \mu$.

3. Région antérieure (céphalon, péréionites libres 1 et $2=$ segments thoraciques 2 et 3 ) de la O adulte de $3,3 \mathrm{~mm}$; $=200 \mu$.

4. Troisième article de la hampe de l'antenne gauche du $\hat{0}$ de $3,2 \mathrm{~mm}$, montrant la squama; $\mathrm{e}=50 \mu$.

5. Péréiopode 1 gauche du $\hat{\delta}$ de $3,2 \mathrm{~mm} ; \mathrm{e}=50 \mu$.

6. Péréiopode 1 droit de la $Q$ à marsupium de $3,2 \mathrm{~mm} ; \mathrm{e}=50 \mu$.

7. Extrémité d'une antennule d'un individu de $1,9 \mathrm{~mm} ; \mathrm{e}=50 \mu$. 
STENASELLUS BRAGAI n. sp.

287

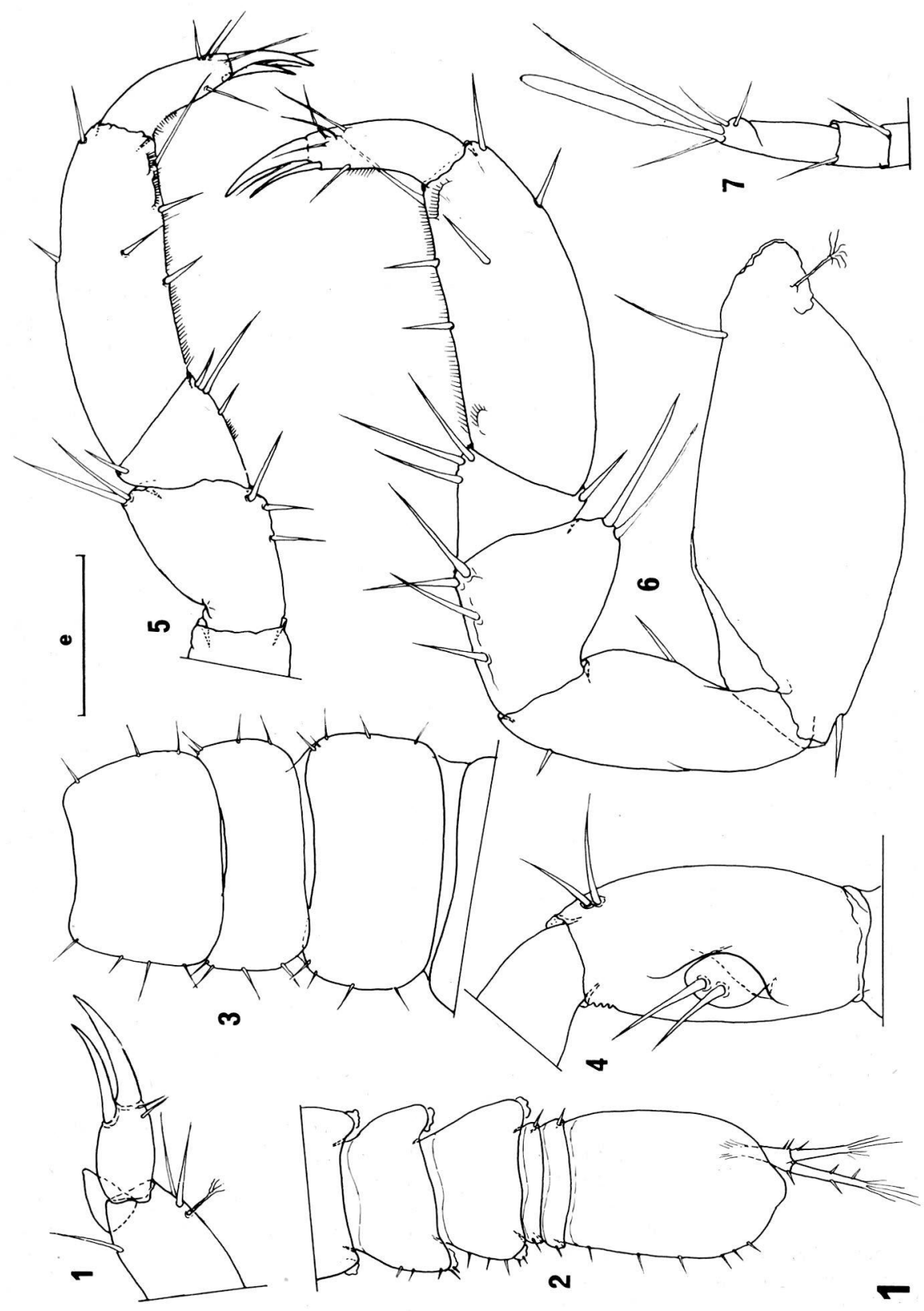


vers l'intérieur; lobe moyen avec 6-7 tiges identiques aux précédentes; lobe interne bifide distalement, portant une dizaine de tiges barbelées, implantées sur deux rangs distaux et de fines soies sur la marge interne.

Maxillipèdes: Typiques de Stenasellidae, sans épipodite, portant un oostégite subréniforme glabre de $370 \mu$ de long et $150 \mu$ de large chez la ò à poche incubatrice. Endite avec 2 crochets rétinaculaires.

Péréiopodes I: Exceptionnellement grêles (tant chez le $\delta$ que chez la $q$, leur diamètre est du même ordre que celui de la hampe de l'antenne), (fig. 5 et 6). Propodite non renflé, avec 2 tiges simples sur la marge sternale. Dactylopodite avec 1 seule tige sternale insérée juste sous l'ongle. Péréiopodes II à VII: Très grêles et fragiles, à chétotaxie très réduite, sauf pour les soies sensorielles de la crête tergale des basipodites. Dactylopodites avec une seule épine sternale subunguéale (fig. 1). Les coxopodites II, III et IV portent de grands oostégites membraneux chez la $q$ à marsupium et des oostégites non fonctionnels en forme de languettes subquadrangulaires, semblables à ceux de $S t$. virei, chez les $q$ adultes au repos génital. Une paire de papilles génitales bien développées insérées à l'angle interne des coxopodites VII chez les $\delta$.

Pléopodes II $q$ : Subtriangulaires, petits et indépendants (fig. 10).

Pléopodes I $\hat{o}$ : Protopodite subquadrangulaire, sans rétinacle, avec des sétules sur la marge latérale; exopodite subquadrangulaire avec une rangée de 4-6 tiges légèrement plumeuses sur la marge interne distale et 3-6 sétules distales (fig. 8).

Pléopodes II $\delta$ : Protopodite subtrapézoïdal avec une forte épine submarginale sternale (fig. 9). Exopodite petit, mais fortement musclé. Endopodite à article proximal long et grêle; article distal en massue, très développé. Un double bombement interne porte de fines soies qui font saillie par l'orifice efférent distal. L'organe copulateur est armé d'une rangée subdistale sternale, puis marginale externe de 7 épines courtes et épaisses (fig. 11).

Pléopodes III: Exopodite (opercule) très allongé; endopodite ovalaire petit et charnu (fig. 12).

Planche II:

8. Pléopode 1 droit du $\hat{o}$ de $2,9 \mathrm{~mm}$; e $=50 \mu$.

9. Pléopode 2 droit du même, face tergale. Les deux lèvres de l'orifice efférent distal de l'organe copulateur ont été artificiellement écartées; $\mathrm{e}=50 \mu$.

10. Pléopode 2 droit de la $q$ à marsupium de $3,2 \mathrm{~mm}$; e $=100 \mu$.

11. Endopodite du pléopode 2 gauche, face sternale, du $\hat{\delta}$ de $3,2 \mathrm{~mm}$. L'organe copulateur n'est pas déformé (comparer avec la fig. 9). L'orifice afférent proximal et la rangée de courtes épines submarginales distales sont visibles; $\mathrm{e}=50 \mu$.

12. Pléopode 3 droit du $\hat{\delta}$ de $2,9 \mathrm{~mm}$; e $=200 \mu$.

13. Pléopode 5 droit de la $q$ à marsupium de $3,2 \mathrm{~mm}$; $\mathrm{e}=100 \mu$.

14. Pléopode 4 gauche du $\hat{\delta}$ de $2,9 \mathrm{~mm}$; e $=200 \mu$.

15. Mandibule gauche du $\delta$ de $3,2 \mathrm{~mm}$; e $=50 \mu$. 


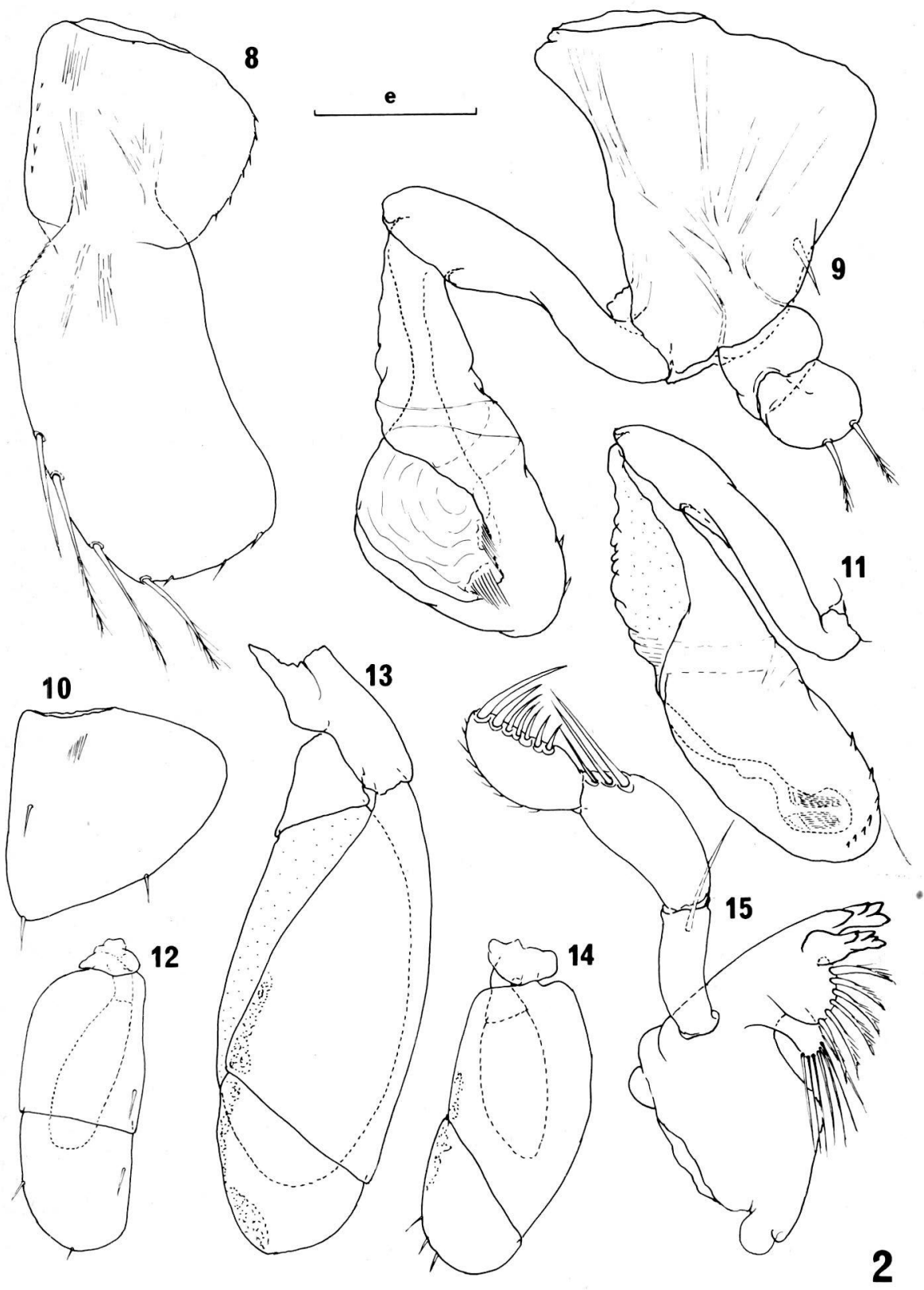


Pléopodes IV: Exopodite lamelleux, long et large, avec suture interarticulaire très oblique; quelques courtes soies marginales distales; endopodite ovalaire, petit et charnu (fig. 14).

Pléopodes V: Exopodite induré, en massue, plus long et aussi large que l'endopodite; une aire glandulaire couverte d'écailles cuticulaires pectinées sur la marge interne-distale; endopodite ovalaire charnu et respiratoire, comme les endopodites III et IV (fig. 13).

Uropodes: biramés normaux, mais bien plus courts que le pléotelson (fig. 2).

\section{Affinités:}

1. Stenasellus bragai $\mathrm{n}$. sp. possède tous les caractères importants qui définissent les Stenasellidae: grand développement des pléonites libres I et II, squama sur A2, mandibule recourbée, maxillipèdes sans épipodite, endopodite des pléopodes II $\hat{o}$ biarticulé, etc.

2. L'architecture du pléopode II $\hat{\delta}$ et des autres pléopodes permet d'inclure cette espèce dans le genre Stenasellus s. str. (cf. Magniez 1974). 3. Les détails de structure (épines distales sur l'organe copulateur. aspect des pléopodes I $\hat{O}$, les pléopodes IV et $\mathrm{V}$ ), montrent qu'il s'agit d'une espèce de la lignée ibérique de St. breuili et St. galhanoge.

4. St. bragai n. sp. est une forme naine, adaptée à la vie psammique. $\mathrm{Du}$ point de vue écologique, on peut la rapprocher d'Asellidae, comme Proasellus walteri (Chappuis), Psammasellus capitatus Braga, Synasellus barcelensis Noodt et Galhano ou de Microparasellidae comme les Microcharon, qui occupent des biotopes de même type.

5. Dans une publication précédente (Magniez 1974), j’ai noté (p. 43) la découverte et (p. 48, fig. 10, $\mathrm{n}^{0} 24$, Stenasellus sp. nov. 1), la localisation géographique de St. bragai n. sp..

\section{RÉSUMÉ}

Cette très petite espèce a été capturée dans la nappe fluviale du Guadalquivir (province de Jaèn). Elle appartient à la lignée de Stenasellus breuili Racovitza, répandue dans les eaux souterraines de la péninsule ibérique.

\section{BIBLIOGRAPHIE}

BRAGA, J. M. 1962. Sur la distribution géographique des Stenasellus de la péninsule ibérique et description d'une espèce nouvelle de ce genre. An. Fac. Ciên. Porto, 44, 9-28.

MAGNIEZ, G. 1968. L'espèce polytypique Stenasellus virei Dollfus, 1897 (Crustacé Isopode hypogé). Ann. Spéléol. Fr.. 23, 363-407.

MAGNIEZ, G. 1974. Données faunistiques et écologiques sur les Stenasellidae (Crustacea Isopoda Asellota des eaux souterraines). Int. I. Speleol., 6, 1-80.

RACOVITZA, E. G. 1950. Asellides, première série: Stenasellus. Arch. Zool. expér. gén. Fr., 87, 1-94. 